Instituto Internacional de Investigación y Desarrollo Tecnológico Educativo INDTEC, C.A.

DOI: https://doi.org/10.29394/scientific.issn.2542-2987.2017.2.6.13.255-269

OAI-PMH: http://www.indteca.com/ojs/index.php/Revista Scientific/oai

\title{
Didáctico Complementario para el Aprendizaje de la Unidad Curricular Mecánica Racional 10
}

\author{
Autores: Pedro Arístides Salas Velázquez \\ Universidad de los Andes, NUAA-ULA \\ pedrosalas@ula.ve \\ Mérida, Venezuela \\ Milagro Yesenia Montilla Davila \\ Universidad de los Andes, NUAA-ULA \\ milagroy@ula.ve \\ Mérida, Venezuela \\ Domingo Alberto Alarcón \\ Universidad de los Andes, NUAA-ULA \\ domingoa@ula.ve \\ Mérida, Venezuela
}

\section{Resumen}

La elaboración del siguiente proyecto educativo inicia con el diagnóstico de las causas del bajo rendimiento de los estudiantes del Núcleo Universitario Alberto Adriani en el estudio de la unidad curricular Mecánica Racional 10. Estas causas fueron determinadas por el investigador a través de una serie de encuestas no estructuras a sus estudiantes durante tres semestres consecutivos. Mediante las diferentes técnicas existentes para la enseñanza y el aprendizaje (tales como la aplicación de la teoría cognitivista, la influencia del paradigma histórico-social en el cual se desenvuelve el estudiante, la aplicación del método inductivo y las diversas estrategias actuales de aprendizaje), se obtuvo una compilación digital de los temas considerados. La asignatura consta de seis unidades de aprendizaje, para cada una de las cuales se desarrollaron los siguientes aspectos: introducción al tema, objetivos formativos que debe aprobar el estudiante, presentación de ejemplos de situaciones reales para mejorar su compresión, breve explicación teórica, elaboración de ejercicios propuestos para ser desarrollados en el salón de clases y en las horas de estudio, referencias bibliográficas y/o electrónicas para consultas fuera del aula de clase.

Palabras clave: didáctica; aprendizaje; enseñanza. 


\title{
Supplementary Didactic Material to Improve the Curriculum Unit of Rational Mechanics 10
}

\begin{abstract}
This educational project begins with the diagnosis of the academic underachievement in Alberto Adriani University core students, specifically in the Rational Mechanics 10 course. A digital compilation was obtained through different techniques which involve learning and teaching processes (the application of cognitive theory, the influence of the historical-social paradigm in which the student develops, the application of the inductive method and the various current strategies of learning). The course consists of six learning units, where the following aspects were developed: theme introduction, learning objectives which students must achieve, real situations examples to improve learning, brief theoretical explanation, proposed exercises for being solved in the classroom in hours of study, bibliographic and electronic references to consult outside the classroom.
\end{abstract}

Keywords: didactics; learning; education. 


\section{Introducción}

El estudio de la ingeniería requiere del conocimiento de asignaturas básicas que ayudarán al estudiante a entender las distintas materias del ciclo profesional. Entre estas materias se encuentra el curso de Mecánica Racional 10 perteneciente al ciclo básico de ingeniería.

La Mecánica Racional 10 es una asignatura que forma parte de la física para el estudio de los cuerpos en reposo sometidos a la acción de fuerzas. Abarca el estudio de las partículas y del cuerpo rígido, necesario para el dominio de materias pertenecientes a la mecánica de sólidos. Las leyes, principios y conceptos fundamentales están fundamentados en la mecánica newtoniana, que requieren de un proceso sistemático para su enseñanza y aprendizaje (Hibbeler R., 2010: 125).

Debido a su importancia académica, el proceso de enseñanza exige que el estudiante valore la importancia del curso de la materia para su formación académica, comprenda los principios fundamentales que se aplican al estudio de la partícula y del cuerpo rígido, defina el concepto de vector en el estudio de la estática y analice los problemas básicos de ingeniería según los conocimientos adquiridos, entre otros. Con la finalidad de ayudar al aprendizaje de estas competencias se desarrolla un material didáctico complementario según ciertos conceptos del proceso educativo.

Por tales razones, este artículo describe a continuación una alternativa en el proceso de enseñanza-aprendizaje de esta asignatura básica de la ingeniería, para que sea analizado y discutido por profesionales de otras universidades con la finalidad de valorar el amplio sentido de responsabilidad docente que debemos aportar en nuestras casas de estudios, orientándonos hacia la formación de un profesional que cumpla cabalmente con las exigencias que la sociedad le pueda demandar. 


\section{Fundamentos teóricos considerados para el proceso de enseñanza y aprendizaje.}

La educación se fundamenta en varias disciplinas de las ciencias humanas, justificando su carácter de ciencia social aplicada, sin desconocer ni dejar de lado su vocación de arte práctico y creativo (PAD, 2010: 20). Esta vocación debe estar orientada de forma que el estudiante desarrolle su espíritu crítico y su creatividad, logrando así un adecuado equilibrio entre su formación técnica-científica y su desarrollo espiritual.

En el transcurrir del tiempo han surgido numerosos fundamentos teóricos que intentan explicar el comportamiento del sujeto, aportando ideas para su enseñanza. Estos fundamentos teóricos se describen como paradigmas educativos y se reconocen universalmente a las siguientes corrientes del pensamiento como las principales: conductismo, cognitivismo, humanismo, constructivismo. Entre estas corrientes, el proceso de aprendizaje requerido para la unidad curricular Mecánica Racional 10 se fundamenta en dos teorías: la cognitivista y el histórico social. Se seleccionan estas dos teorías por las siguientes razones:

- Se requiere que el estudiante organice los conocimientos a adquirir por medio de esquemas y experiencias que puedan formar parte de su memoria implícita a través de su atención y la discusión de ideas que enriquezcan su manera de percibir el mundo mediante el raciocinio (Cognitivismo).

- La situación económica actual, ya que muchos estudiantes no disponen de los recursos suficientes para la adquisición de material de apoyo (tal como computadoras, libros y calculadoras de altas tecnología). Esto determina que se tome en cuenta la realidad social para no interferir en la calidad educativa (Paradigma histórico-social). 
No basta con definir estas teorías pues todo proceso educativo requiere, por lo menos, de los modelos de enseñanza y de las estrategias de aprendizaje (Salas P., 2012: 35). Los modelos de enseñanza se pueden clasificar según las formas de razonamiento en: método analítico, método analógico o comparativo, método inductivo y método deductivo (PAD, 2010: 35). La definición y estudio de estos conceptos de razonamiento hacen posible que la enseñanza de la Mecánica Racional 10 sea apoyada por el método inductivo. Es decir, se requiere que el facilitador debe inferir en el nivel de abstracción de sus estudiantes para que, de esta manera, evalúe las competencias propuestas en cada una de las unidades de aprendizaje. Por otro lado, las estrategias de aprendizaje que se definen como las actividades que el estudiante realiza con la finalidad de aprender (Campos Y., 2000: 125) deben ser analizadas según el entorno social del educando para que logre aprobar las diferentes competencias y objetivos contemplados en su contenido programático.

Estos breves conceptos se consideran suficientes para la elaboración del material de apoyo educativo de la asignatura. Teniéndose en cuenta que la discusión de los resultados alcanzados permitirá que otros profesionales en la enseñanza de esta unidad curricular debatan acerca de mejoras a esta iniciativa para contribuir a que el estudiante sienta un mayor interés de alcanzar las competencias necesarias para su aprendizaje.

\section{Desarrollo del material didáctico y complementario}

Considerándose que este trabajo no es un diseño instruccional de la unidad curricular en estudio, se ha tenido en cuenta ciertas concepciones relacionadas con la planificación del proceso instruccional y los elementos que deben estar presentes de forma sistemática y organizada en la práctica educativa. Esto permite que el material elaborado tenga el soporte metodológico e investigativo adecuado a las exigencias necesarias para que 
se logre los objetivos planteados a través de un desarrollo de ciertas etapas, organizadas sistemáticamente durante 16 semanas de investigación:

- Etapa 1. Diagnóstico del bajo rendimiento de los estudiantes del Núcleo Universitario Alberto Adriani para el aprendizaje de la unidad curricular Mecánica Racional 10. En esta etapa el investigador cuenta con los datos obtenidos previamente de encuestas no estructuradas a sus estudiantes durante un periodo de tres semestres académicos (48 semanas).

- Etapa 2. Recopilación de la información. En esta etapa se depuraron los datos obtenidos del diagnóstico. Además, se desarrolló la revisión bibliográfica y/o electrónica de material de aprendizaje y la disposición de técnicas y recursos para el desarrollo del proyecto.

- Etapa 3. Elaboración del anteproyecto. Se expone ante un jurado el planteamiento del problema, la justificación de la investigación, los alcances y limitantes encontradas y una descripción general del material desarrollado por el autor.

- Etapa 4. Ejecución del proyecto. En esta etapa se desarrolla todo el material didáctico escogido para el proceso de enseñanza-aprendizaje de la asignatura.

- Etapa 5. Elaboración del producto final. El proyecto desarrollado consistirá en un informe de cómo se realizó la investigación, la elaboración de guías de estudio, diapositivas para exposición de los temas y/u otro material que se consideren necesarias.

- Etapa 6. Presentación del producto final. Luego de haberse discutido con el jurado el alcance de la investigación y su valoración académica dentro del proceso de enseñanza-aprendizaje se sugiere la presentación del artículo a una revista de investigación reconocida a fin 
de que lo expuesto sea analizado y discutido por otros grupos de investigación en educación de ingeniería.

El proceso comienza con la definición de la unidad curricular dentro del programa de estudio en la Universidad de Los Andes. Luego, se describe brevemente un diagnóstico del bajo rendimiento de los estudiantes en la aprobación inmediata de la asignatura, fundamentándose en encuestas no estructuras realizadas de manera oral por el investigador a sus estudiantes durante su tiempo de experiencia docente. Luego, se desarrolla de manera sistemática la elaboración de material multimedia (diapositivas en Microsoft PowerPoint) para el dictado de clases y del desarrollo de guías de estudios como material complementario; considerándose en ambos casos los objetivos formativos exigidos en cada unidad de aprendizaje.

La unidad curricular Mecánica Racional 10 es un curso fundamental de carácter formativo en conceptos y principios de la mecánica del cuerpo rígido en áreas de la ingeniería, Civil, Geológica y Mecánica (Ver tabla 1). Cuya justificación en el proceso de formación del ingeniero es aportar los conocimientos necesarios en la aplicación de las leyes de Newton para la estática de los cuerpos rígidos, necesaria para la compresión de cursos posteriores en Mecánica de Materiales, Estructuras y Elementos de Máquinas. El contenido programático está constituido por seis (6) unidades de aprendizaje (U.L.A., 2001: 3):

- Unidad de aprendizaje I. Estática de las partículas.

- Unidad de aprendizaje II. Cuerpos rígidos.

- Unidad de aprendizaje III. equilibrio.

- Unidad de aprendizaje IV. Reticulados.

- Unidad de aprendizaje V. Fuerza cortante y momento flector.

- Unidad de aprendizaje VI. Propiedades de áreas planas. 
Tradicionalmente, esta asignatura presenta un alto porcentaje de estudiantes aplazados y requiere de una o más veces ser vista por el estudiante para poder aprobar sus objetivos formativos. Esto ha llevado al investigador a realizar una serie de encuestas no estructuras a sus estudiantes durante tres semestres consecutivos para diagnosticar la falta de motivación en la materia. Entre las causas que originan desmotivación en el proceso de aprendizaje se encuentran:

- Carencia de ejemplos prácticos reales cuando se imparte las clases presenciales.

- Pocos ejemplares de libros en la biblioteca para poder realizar préstamos externos.

- Lenguaje técnico avanzado en aquellos libros que el docente sugiere para la consulta.

- El estudiante no entiende coloquialmente los objetivos que persigue la materia en su formación profesional.

- El estudiante no está claro si la ingeniería que escogió para su vida profesional es la que quiere estudiar. Esto hace que al encontrarse con una materia de análisis que requiere de la aplicación de técnicas cognitivas para su aprendizaje no sea exitosamente desarrollado.

Tabla 1. Identificación de la asignatura Mecánica Racional 10.

\begin{tabular}{|c|c|c|}
\hline $\begin{array}{c}\text { Unidad Curricular: } \\
\text { Mecánica Racional 10 }\end{array}$ & $\begin{array}{c}\text { Código: } \\
1019 / 1067\end{array}$ & $\begin{array}{c}\text { Unidades de crédito: } \\
\text { Régimen: }\end{array}$ \\
$\begin{array}{c}\text { Modalidad: } \\
\text { Presencial }\end{array}$ & $\begin{array}{c}\text { Carácter: } \\
\text { Obligatorio }\end{array}$ \\
\hline $\begin{array}{c}\text { Semestre de Ubicación: } \\
\text { Tercer Semestre }\end{array}$ & $\begin{array}{c}\text { Carreras: } \\
\text { Ingenierías: Mecánica, Civil, Geológica }\end{array}$ \\
\hline
\end{tabular}




\begin{tabular}{|c|c|c|}
\hline $\begin{array}{l}\text { Prelación: } \\
\text { Cálculo 20/ Física } 11\end{array}$ & $\begin{array}{c}\text { Horas de } \\
\text { clases/semestre: } 90\end{array}$ & $\begin{array}{c}\text { Horas de } \\
\text { clases/semana: } 5\end{array}$ \\
\hline $\begin{array}{c}\text { Densidad horaria semanal: } \\
\text { Horas Teóricas: } 4 \text { Hora } \\
\text { Prácticas: } 1 \text { Horas }\end{array}$ & $\begin{array}{r}\text { Vigencia: } \\
\text { Se }\end{array}$ & stre B-2001 \\
\hline
\end{tabular}

Fuente: Coordinación Académica Núcleo Universitario Alberto Adriani (N.U.A.A. 2001).

Estas causas motivaron a que se organizara sistemáticamente un material didáctico complementario para el aprendizaje de esta asignatura a través de los modelos de enseñanza y de las estrategias de aprendizaje que motiven al estudiante a tomar interés en valorar la importancia del curso de Mecánica Racional 10. Este material contiene información de ayuda para el proceso de enseñanza-aprendizaje como la siguiente:

- Descripción detallada de los objetivos formativos que el estudiante debe lograr en cada tema a evaluar.

- Desarrollo de los fundamentos teóricos necesarios para cada unidad de aprendizaje considerándose un lenguaje más coloquial para ser leído y discutido.

- Visualización gráfica del material en su computadora con diseño de colores e imágenes pertinentes a la edad de los estudiantes para que se sienta motivado.

- Clasificación de los ejercicios a desarrollar en clases y en casa para que aborde puntos esenciales del proceso de aprendizaje. 


\section{Impacto que pueda generarse en el proceso de enseñanza y aprendizaje.}

El material desarrollado de forma didáctica y complementaria a la información suministrada por el docente y los libros texto guías, ofrecen ciertas ventajas en el proceso de enseñanza-aprendizaje. Primeramente, permite que el docente pueda interactuar de manera más animada con sus estudiantes en el salón de clases. Seguidamente, facilita la planificación y la evaluación de los objetivos formativos para lograr las competencias exigidas. Por otro lado, ayuda al estudiante a que sea más fácil la organización de su metodología de estudio. Y, aunque el libro texto es esencial para la compresión de la unidad curricular, el material complementario ayudará a que el estudiante puede entender más fácilmente la información técnica. Sin embargo, hay que estar consciente que proporcionar material de apoyo al estudiante para la compresión de la asignatura, puede crearles vicios en su desarrollo educativo al evitarle consultar textos de interés. Por lo tanto, se recomienda tener mucho cuidado en no proporcionar toda la información para que puedan verse obligados a consultar las respectivas referencias bibliográficas necesarias para profundizar sus conocimientos. Esto quiere decir, que el docente debe tener presente que la información que suministre a sus alumnos debe ser cíclica. Primero, impartirá sus clases con material didáctico y su experiencia. Luego, sugerirá a sus alumnos que revisen el material complementario proporcionado para poder leer el texto guía. Finalmente, el estudiante a través de actividades guiadas fuera del aula de clases se verá en la obligación de revisar las bibliografías pertinentes y de asistir a las consultas tutoradas por el profesor con la finalidad de aclarar términos y dudas.

De esta manera, el docente logrará que el proceso de aprendizaje sea cognitivo y forme parte del proceso histórico-social del estudiante al adecuarse a las nuevas inquietudes del entorno educativo de nuestra sociedad. 


\section{Conclusión}

La elaboración del material didáctico complementario para la unidad curricular Mecánica Racional 10 permitirá que los estudiantes de ingeniería rompan los paradigmas que poseen de la asignatura, la cual consideran de poca valoración por ser su aprendizaje tedioso, dificultoso, extenso y abstracto. Esto no quiere decir que se pretenda sustituir la enseñanza que el profesor realiza en su aula de clase por enseñanza a distancia y autodidáctica, ni tampoco que es un material realizado a la perfección. La idea del proyecto es lograr alcanzar la mejora continua en el aprendizaje de los estudiantes con recursos de apoyo que siempre requerirán de la experiencia del docente en la unidad curricular que imparte. Por lo tanto, se recomienda la revisión continua del diseño instruccional, la adaptación de las estrategias en la metodología instruccional según el contexto histórico-social de los estudiantes y la discusión permanente de ideas para el proceso de enseñanza y aprendizaje. Todo dirigido a entender que el estudiante cumple un rol social según la comunidad en la que se desenvuelve desde su etapa de iniciación como profesional.

\section{Referencias}

Campos Y. (2000). Estrategias didácticas apoyadas en tecnología. DGENAMDF: México. Recuperado de: http://files.estrategias2010.webnode.es/200000040042b505253/estrategias\%20didactica\%20y\%20la\%20tecnologia.pdf Hibbeler R. (2010). Mecánica Vectorial para ingenieros. Estática. 9ª edición. México: Editorial McGraw-Hill.

Programa de Actualización de los Docentes (PAD, 2010). Diseño Instruccional. Componente Docente Básico en Educación superior. Mérida: Vicerrectorado Académico de la Universidad de Los Andes. Venezuela. 
Salas P. (2012). Diseño instruccional de la unidad curricular resistencia de materiales. Trabajo Especial del Componente Docente Básico en Educación Superior. Programa de actualización de docente. Componente docente básico en educación superior. Mérida: Universidad de Los Andes. Venezuela.

U.L.A. (2001). Programa de estudios de la asignatura Mecánica Racional 10. Escuela Básica de Ingeniería. Mérida: Universidad de Los Andes. 
Pedro Arístides Salas Velázquez

e-mail: pedrosalas@ula.ve

Nacido en Tovar estado Mérida, Venezuela. Egresado

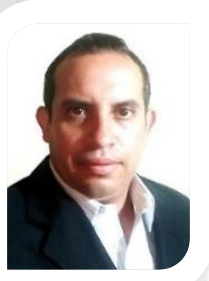
como Ingeniero Mecánico en la Universidad Nacional Experimental del Táchira (U.N.E.T) en el año 2005. con Realiza estudios de Maestría en Ingeniería de Mantenimiento en la Universidad de Los Andes (U.L.A.) en el año 2010. Actualmente, finaliza trabajo de grado en la Maestría de Ingeniería Mecánica, mención Diseño y Manufactura de la U.L.A. En el año 2012 realiza el Componente Docente Básico en Educación Superior. Se desempeña en la actualidad como Docente Asistente de la Universidad de Los Andes adscrito al Núcleo Universitario Albero Adriani en la ciudad del Vigía, Mérida Venezuela. 


\section{Milagro Yesenia Montilla Dávila}

e-mail: milagroy@ula.ve

Nacida en el Vigía Edo. Mérida, Venezuela.

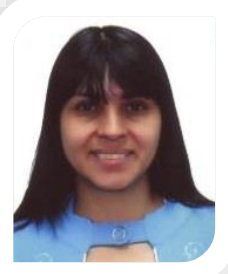
Actualmente realizo estudios de Doctorado en Ciencias de la Educación en la Universidad Fermín Toro, Barquisimeto Edo. Lara. Egresada de la Universidad de los Andes (ULA) como Licenciada en Química, Magister en Electroquímica Fundamental y Aplicada (ULA); estoy realizando labores de Docencia en el Área de Química para las carreras de Ingeniería (ULA); actualmente soy la coordinadora Académica del NUAAULA e investigadora Activa de la Universidad de los Andes, Programa de Estímulo a la Investigación PEI-ULA y del Ministerio del Poder Popular para la Educación Universitaria, Ciencia y Tecnología PEII-ONCTI; he participado en el dictado de talleres, organización de Jornadas para estudiantes universitarios de nuevo ingreso, ponente Nacional e Internacional. 
Nacido en Tovar Edo. Mérida, Venezuela.

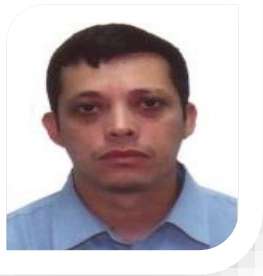
actualmente realizo estudios de Doctorado en Ciencias de la Educación en la Universidad Fermín Toro, Barquisimeto Edo. Lara. Egresado de la Universidad de los Andes (ULA) como Licenciado en Química, Magister en Electroquímica Fundamental y Aplicada (ULA); estoy realizando labores de Docencia en el Área de Química para las carreras de Ingeniería (ULA); actualmente soy Director del NUAAULA, Coordinador General del Grupo de Investigación Interdisciplinario en Ingeniería y Educación GIIIE e investigador Activo de la Universidad de los Andes, Programa de Estímulo a la Investigación PEI-ULA y del Ministerio del Poder Popular para la Educación Universitaria, Ciencia y Tecnología PEllONCTI; he participado en el dictado de talleres, organización de Jornadas para estudiantes universitarios de nuevo ingreso, ponente Nacional e Internacional.

El contenido de este manuscrito se difunde bajo una Licencia de Creative Commons ReconocimientoNoComercial-Compartirlgual 4.0 Internacional 